\title{
Effect of closed incision negative pressure wound therapy on incidence rate of surgical site infection after stoma reversal: a pilot study
}

\author{
Maciej Borejsza-Wysocki ${ }^{1}$, Adam Bobkiewicz ${ }^{1}$, Wojciech Francuzik², Lukasz Krokowicz ${ }^{1}$, Dominik Walczak ${ }^{3}$, \\ Jacek Szmejaㅁ, Tomasz Banasiewicz ${ }^{1}$ \\ ${ }^{1}$ Department of General, Endocrinological and Gastroenterological Oncology Surgery, Poznan University of Medical Sciences, Poznan, \\ Poland \\ 2Department of Dermatology, Venerology and Allergology Charité - Universitätsmedizin, Berlin, Germany \\ ${ }^{3}$ Department of Oncological and Reconstructive Surgery, Maria Sklodowska-Curie National Research Institute of Oncology, Gliwice, \\ Gliwice, Poland
}

Videosurgery Miniinv 2021; 16 (4): 686-696 DOI: https://doi.org/10.5114/wiitm.2021.106426

\begin{abstract}
Introduction: The stoma reversal (SR) procedure is associated with a relatively high risk of perioperative complications with surgical site infection (SSI) as the most common. Recently closed incision negative pressure wound therapy (ciNPWT) was applied widely to prevent SSI.

Aim: To investigate the efficiency of ciNPWT in terms of the incidence rate of SSI after SR surgery.

Material and methods: As an exploratory observational cohort study patients were treated either with ciNPWT $(n=15)$ or standard sterile dressing (SSD) $(n=15)$. CiNPWT was applied every 3 days whereas SSD was changed every day. Clinical evaluation for SSI signs, C-reactive protein level and pain assessment using the visual analogue scale (VAS) were analyzed.

Results: The incidence rate of SSI was in 13\% (2/15) in the ciNPWT group and 26\% (4/15) in the SSD group ( $p=0.651, O R=0.44,95 \% \mathrm{Cl}: 0.03-3.73)$. All patients in the SSD group who developed SSI presented both local and generalized signs of infection. Pain-VAS levels assessed on the $1^{\text {st }}(M d n c i N P W T=4, M d n S S D=5, p=0.027, W=$ 51.5 ) and $3^{\text {rd }}$ postoperative day (MdnciNPWT $=2, M d n S S D=4, p=0.014, W=45.5$ ) were significantly lower in the ciNPWT group than in the SSD group.

Conclusions: CiNPWT seems not to have a benefit to reduce SSI after the SR procedure. Further investigation is needed to establish firmly the benefit of using ciNPWT in this group of patients.
\end{abstract}

Key words: closed incision negative pressure wound therapy, stoma reversal, surgical site infection.

\section{Introduction}

Currently, anal sphincter-preserving surgery is one of the main goals in colorectal surgery. However, defunctioning ileostomy is widely recommended in varying clinical scenarios such as low rectal resection or restorative proctocolectomy $[1,2]$.
The stoma reversal procedure is associated with perioperative complications classified as grade I or grade II according to the classification of surgical complications proposed by Dindo et al. and may affect up to $40 \%$ of patients $[3,4]$. Moreover, a recent systematic review showed an overall mortality rate of $0.4 \%$ due to 
stoma reversal procedures [5]. Surgical site infection (SSI) is the most common and burdensome complication after stoma reversal (SR) surgery. The incidence rate of SSI after stoma reversal ranged from $2 \%$ to $41 \%$ [6]. However, introduction of pursestring closure technique to surgical practice resulted in a significant decrease of the surgical site infection rate after the SR procedure [7]. Hsieh et al. in a meta-analysis of randomized controlled trials revealed that pursestring closure had significantly fewer surgical site infections in contrast to conventional primary closure. However, it was limited to the lack of double blinding and longterm follow-up in the included analyses.

Since negative pressure wound therapy (NPWT) was introduced for commercial use in the early 1990s, the strategy of wound management has been revolutionized. The mechanism of action includes reduction of interstitial edema, stimulation of granulation tissue, mechanical wound cleansing, increased microcirculation and tissue oxygenation, decreased wound area and others, which accelerate wound healing [8]. Currently, NPWT is used in varying clinical scenarios, different medical indications and affected areas of the human body. Recently, closed incision NPWT (ciNPWT) was introduced to prevent surgical site infection (SSI). CiNPWT significantly reduced the incidence rate of SSI after sternotomy [9], hip and knee arthroplasties [10], in colorectal patients $[11,12]$ and Crohn's disease patients [13], in a groin vascular procedure [14] and spinal surgery [15]. Recently, Poehnert et al. [16] and Uchino et al. independently investigated the impact of ciNPWT on the incidence of surgical site infection after the stoma reversal (SR) procedure [17]. However, the outcomes of those two studies are conflicting. Thus, there is a need for further clarification of the indication of using ciNPWT in the SR procedure.

The risk of transmission of endogenous bacteria into the incision line may result in surgical site infection. Although new strategies and devices were introduced to routine practice such as antibiotic-coated sutures, silver-impregnated dressings, cold-plasma scalpels, and iodine-impregnated skin drapes, the cumulative risk of SSI in stoma reversal procedures is still relatively high.

\section{Aim}

The aim of the present study was to investigate the efficiency of portable, no-canister containing incisional negative pressure wound therapy (PICO, Smith \& Nephew Ltd, UK) on the incidence rate of SSI after SR surgery.

\section{Material and methods}

The study was approved by the institutional bioethics committee at Poznan University of Medical Sciences (trial number 533/14). The study was conducted between May 2015 and January 2017. A total of 30 patients who underwent stoma closure were treated either with NPWT (NPWT group) or standard sterile dressing (SSD group). The study was designed as a randomized, prospective, explorative, cohort observational superiority study.

\section{Objectives}

The primary objective was to assess the difference in the incidence rate of SSI between ciNPWT and SSD wound care groups.

The secondary objective was to evaluate other surgical site complications (SSC) such as: 1) wound dehiscence rate, 2) seroma and hematoma formation and 3) self-reported pain level as well as to assess the re-admission rate in the analyzed group of patients.

\section{Inclusion and exclusion criteria}

All patients included in the study met the following criteria: 1) written consent for study participation; 2) age above 18 years; 3) defunctioning ileostomy created due to either: a) inflammatory bowel disease, b) colorectal surgery, c) familial adenomatous polyposis or d) iatrogenic complications of small/large bowel; 4) stoma closure procedure performed with stoma site approach (without any other incisions); 5) stoma created at least 3 months prior to inclusion in the study.

Patients who participated in another trial or underwent surgery urgently were not included in this study. Another exclusion criterion was the presence of signs of sepsis as well as coagulopathy diagnosed preoperatively.

\section{Study design}

After informed consent eligible patients were randomly allocated preoperatively either to the ciNPWT group or the standard sterile dressing (SSD) group by using a closed envelope ran- 
domization method. During hospital stay patients were evaluated according to the following schedule - dependent on the dressing changes: 1) ciNPWT: every 3 days or earlier in the case of an unsealed system or layer pad absorbed entirely with wound exudates (a total of three ciNPWT changes were done for every patient; two ciNPWT dressing changes during hospital stay and the third at the time of patient discharge) or 2) SSD: daily dressing changes. The clinical evaluation of SSI signs was performed every day. C-reactive protein (CRP) and the visual analogue scale (VAS) were assessed on the $1^{\text {st }}, 3^{\text {rd }}$ and $5^{\text {th }}$ day.

Follow-up was standardized with visits on the $30^{\text {th }}$ day postoperatively in the outpatient clinic or surveyed via phone call to assess any possible complications.

\section{Data inclusion}

Data were collected based on the available medical records and surgical charts for age, sex, underlying pathology, time interval from stoma creation to stoma reversal, use of steroids and comorbidities, operative time of stoma closure and length of incision line, time of postoperative hospital stay, readmission and mortality rate.

\section{Diagnosis of SSI}

According to Centers for Disease Control and Prevention, superficial SSI was defined in case of skin and subcutaneous tissue infection presenting with pain, redness, heat or swelling at the site of the incision site and/or purulent discharge [18].

\section{Methods of assessment}

The surgical wound was evaluated daily in the control group and every third day in the ciNPWT group when the occlusive dressing was changed for signs of SSI (mentioned above). In the case of SSI, the wound was opened at the site of the highest aggravation of SSI and wound exudate was cultured. The incision line was evaluated clinically in regards to seroma or hematoma presence as well as dehiscence. If needed, ultrasound scan examination was made to assess the hematoma/seroma presence.

Postoperative pain was assessed using the visual analogue scale (VAS). Briefly, patients specified the amount of pain they experienced on the 10-centimeter line. The pain was graded as follows: 0 - no pain,
10 - unbearable pain. Pain VAS rating was evaluated on the $1^{\text {st }}, 3^{\text {rd }}$ and $5^{\text {th }}$ postoperative day.

Patients were tested for CRP level on $1^{\text {st }}, 3^{\text {rd }}$ and $5^{\text {th }}$ day postoperatively. Analysis of CRP level was performed with a Cobas 6000 analyser (Roche Diagnostics, Switzerland) using immunoturbidimetry method. The reference range of CRP level was between 0.01 and $4 \mathrm{mg} / \mathrm{l}$.

\section{Surgical technique}

As antibiotic treatment with cefazoline in a dosage of $2.0 \mathrm{~g}$ was routinely used $1 \mathrm{~h}$ before skin incision, treatment was prolonged over the $24 \mathrm{~h}$ following the surgery. All surgical procedures were performed with the stoma site approach. There were no additional incisions or other surgical approach made for the stoma reversal procedure. Following blunt and sharp dissection the ileal loop was completely separated from the abdominal wall. Then, the skin around the intestine was resected, and margins of the intestine were refreshed and sutured using Monosorb 3/0 (Yavo Medical Supplies Manufacturer, Belchatow Poland). In both groups a handsewn end-to-end anastomosis was performed. The peritoneum, as well as the rectus fascia, was closed in layers using PGLA LACTIC 2 (Yavo Medical Supplies Manufacturer, Belchatow Poland). The wound was irrigated with octenidine dihydrochloride (Octenisept, Schülke \& Mayr GmbH, Germany) and afterwards rinsed with Ringer's solution or $\mathrm{NaCl}$ and the skin was cleansed with povidone-iodine solution (Braunol, BBraun, Melsungen, Germany).

In both groups, the skin was closed primarily with interrupted Nylon 3/0 (Yavo Medical Supplies Manufacturer, Belchatow Poland) in the standard manner. After skin closure, the patient received either ciNPWT dressing or SSD. Standard sterile dressing (gauze and plaster) was applied in the SSD group, whereas in the ciNPWT group, the PICO $10 \times 20 \mathrm{~cm}$ (Smith \& Nephew Ltd, UK) was used (Photo 1). The standard sterile dressing was changed daily by a colorectal nurse and the wound was cleansed with octenidine dihydrochloride solution (Octenisept, Schülke \& Mayr GmbH, Germany). CiNPWT dressing was changed every 3 days or earlier in the case of an unsealed system or insufficiency of the soaking pad. Usually, PICO dressing was changed twice postoperatively. At the time of discharge, a third change of NPWT was routinely done. 


\section{End-point}

The primary end-point of the study was completed on the $30^{\text {th }}$ postoperative day. Appropriate healing was defined macroscopically as proper scar tissue formation at the site of the stoma reversal without signs of inflammation and infection.

\section{Statistical analysis}

The Shapiro test was used to verify normal distribution. We used the Wilcoxon rank sum test with continuity correction (Mann-Whitney $U$ test) when we compared non-normally distributed variables. Wherever categorical variables were compared, we used Fisher's exact test, to account for the low number of observations per group. Results with a $p$-value $<0.05$ were considered statistically significant. The character of this study involving 30 participants, focused on obtaining first insight of the effects of ciNPWT on stoma-reversal wounds, was preliminary (underpowered due to low cohort size) to provide data for further clinical trials.

\section{Results}

Thirty consecutive patients with a median age of $34.5, I Q R=24.5$ (range: $19-68$ ) years were enrolled in the study. Patients were treated ( $n=15$ per group) either with ciNPWT or SSD. The study groups comprised 14 (46.7\%) females and $16(53.3 \%)$ males. The female-to-male ratio was 1.14 and 0.67 , respectively for ciNPWT and SSD groups. The mean body mass index (BMI) was $22.7 \pm 4.7$ and $25.9 \pm 4.4$, respectively for ciNPWT and SSD wound care $(\mathrm{t}(28)=-1.98$, $p=0.058, d=-0.72$ ). All details of demographic and

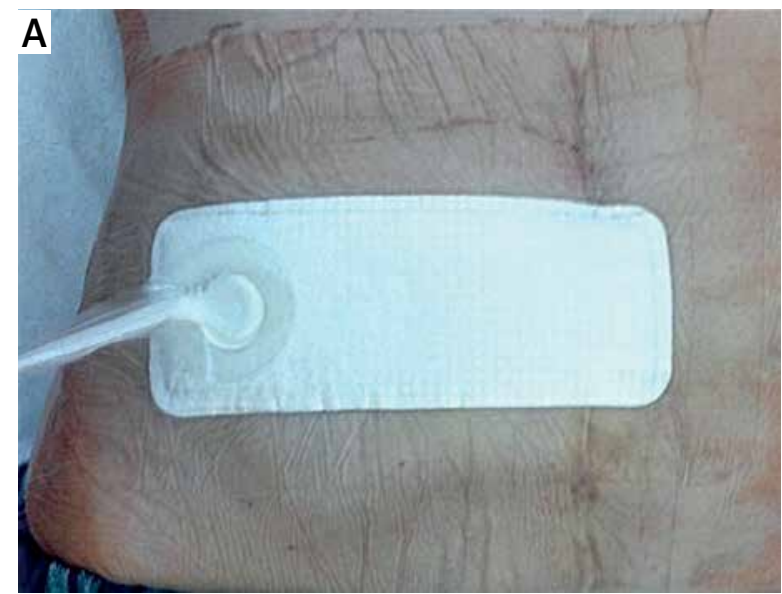

Photo 1. Application of wound dressing in ciNPWT group (A) and standard sterile dressing (SSD) (B)

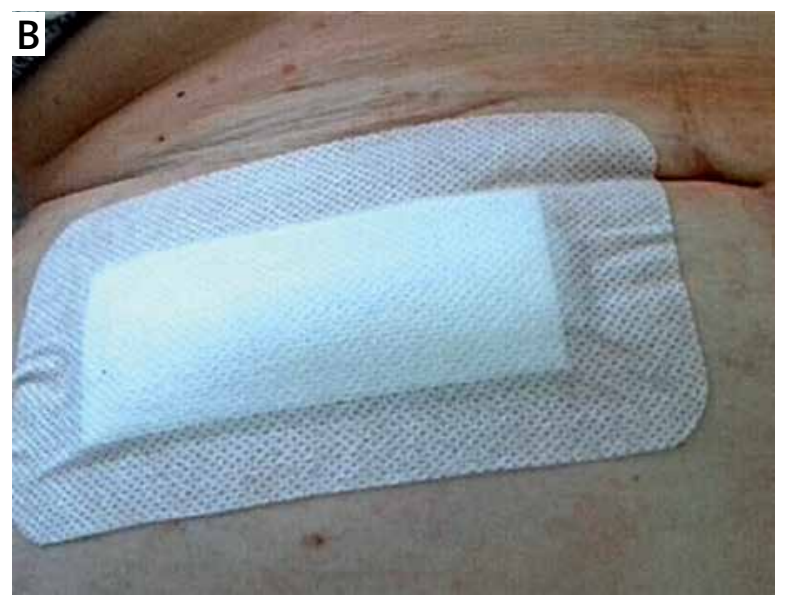

clinical aspects are summarized in Tables I and II, respectively. Progress in wound healing in both study groups on days 0, 3 and 6 are presented in Photo 2 .

Ulcerative colitis was the most common underlying pathology for stoma creation in the ciNPWT and SSD group of patients ( $80 \%$ and $53.3 \%$, respectively). Other indications for stoma creation during index surgery were: familial adenomatous polyposis, colorectal cancer and iatrogenic perforation (during colonoscopy). The steroid regimen was discontinued $M d i=16, I Q R=22$ and $M d i=16.5, I Q R=9.75$ months before the stoma reversal procedure for the ciNPWT group and SSD group, respectively ( $W=$ $52.5, p=0.65,95 \% \mathrm{Cl}:-13.99996-5.00006)$. There was no statistically significant difference in duration from stoma creation to stoma reversal between the ciNPWT group and the SSD group (MdiciNPWT $=11$, IQRCiNPWT $=3.5$ months, $M$ diSSD $=6, I Q R S S D=$ 4 months, $W=153, p=0.09$ ). Smoking was reported by $26 \%$ and $20 \%$ of patients, respectively, for ciNPWT and SSD groups ( $p=1,95 \% \mathrm{Cl}$ : $0.163-$ 10.234). The mean length of hospital stay before surgery was 6 days $(I Q R=2$ and $M d i=4$ days, $I Q R$ $=3.5)$, respectively for ciNPWT and SSD $(W=174.5$, $p=0.018$ ). There was no significant difference in the mean surgery duration time for ciNPWT (Mdi $=70, I Q R$ $=22.5)$ and SSD $(M d i=70, I Q R=20)(W=127.5, p=$ $0.55)$ or in the mean length of incision line ( $M d i=7$, $I Q R=1.5$ and $M d i=7, I Q R=2)$ for ciNPWT and SSD, respectively ( $W=105.5, p=0.78$ ). We did not find any significant differences analyzing CRP level assessed on the $1^{\text {st }}(W=84.5, p=0.41), 3^{\text {rd }}(W=64$, $p=0.50)$ and $5^{\text {th }}(W=77, p=0.52)$ postoperative days between study groups. However, VAS assessed 
Table I. Patient characteristics

\begin{tabular}{|c|c|c|c|}
\hline Parameter & ciNPWT & Standard sterile dressing & $P$-value \\
\hline No. of patients & 15 & 15 & 1 \\
\hline Female/male ratio & $1.14(8 / 7)$ & $0.67(6 / 9)$ & 0.4642 \\
\hline BMI & $22.7 \pm 4.7$ & $25.9 \pm 4.4$ & 0.0579 \\
\hline \multicolumn{4}{|l|}{ Underlying pathology: } \\
\hline Ulcerative colitis & $12(80 \%)$ & $8(53.3 \%)$ & 0.2451 \\
\hline FAP & $1(6.7 \%)$ & 0 & 1 \\
\hline$C R C$ & 0 & $3(20 \%)$ & 0.2241 \\
\hline latrogenic & $2(13.3 \%)$ & $4(26.7 \%)$ & 0.6513 \\
\hline $\begin{array}{l}\text { Steroid regimen discontinued } \\
\text { before stoma reversal [months] }\end{array}$ & $20.7 \pm 6.3$ & $17.9 \pm 5.8$ & 0.6496 \\
\hline $\begin{array}{l}\text { Duration from stoma creation to } \\
\text { stoma reversal [months] }\end{array}$ & $9.8 \pm 3.2$ & $7.8 \pm 3.6$ & 0.09408 \\
\hline Smoking & 4/15 (26.7\%) & $3 / 15$ (20\%) & 1 \\
\hline
\end{tabular}

FAP - familial adenomatous polyposis, CRC - colorectal cancer.

Table II. Specifications of perioperative factors

\begin{tabular}{|c|c|c|c|}
\hline Parameter & NPWT & Standard sterile dressing & $P$-value \\
\hline Inpatient stay before surgery [days] & $6.4 \pm 1.6$ & $4.4 \pm 3.2$ & 0.01018 \\
\hline Duration of surgery [min] & $80.3 \pm 25.7$ & $74.7 \pm 22.9$ & 0.5445 \\
\hline Length of incision line [cm] & $6.8 \pm 1.5$ & $6.9 \pm 1.3$ & 0.7816 \\
\hline \multicolumn{4}{|l|}{ VAS (0-10): } \\
\hline $1^{\text {st }}$ day & $4 \pm 1.1$ & $4.9 \pm 0.9$ & 0.02749 \\
\hline $3^{\text {rd day }}$ & $2.5 \pm 0.9$ & $3.5 \pm 0.9$ & 0.01378 \\
\hline $5^{\text {th }}$ day & $2.3 \pm 1.1$ & $2.9 \pm 0.9$ & 0.1526 \\
\hline \multicolumn{4}{|l|}{ CRP [mg/l]: } \\
\hline $1^{\text {st }}$ day & $28.3 \pm 24.4$ & $15.9 \pm 13.8$ & 0.4123 \\
\hline $3^{\text {rd }}$ day & $46.3 \pm 44$ & $63.6 \pm 68.9$ & 0.5007 \\
\hline $5^{\text {th }}$ day & $22.9 \pm 20.9$ & $28.3 \pm 27.8$ & 0.5186 \\
\hline Inpatient stay after surgery [days] & $7.9 \pm 1.7$ & $7 \pm 2.2$ & 0.06681 \\
\hline SSI & 2/15 (13.3\%) & $4 / 15(26.7 \%)$ & 0.6596 \\
\hline Day of occurrence (mean) & 4 & 4 & \\
\hline \multicolumn{4}{|l|}{ Signs: } \\
\hline Local & $2 / 2$ & $4 / 4$ & 1 \\
\hline Generalized & $1 / 2$ & $4 / 4$ & 1 \\
\hline Hematoma & $0 / 15$ & $4 / 15$ & 0.113 \\
\hline Wound dehiscence & $0 / 15$ & $2 / 15$ & 0.4859 \\
\hline Re-admission rate & $0 / 15$ & $0 / 15$ & 1 \\
\hline Mortality rate & $0 / 15$ & $0 / 15$ & 1 \\
\hline
\end{tabular}

VAS - visual analogue scale. 
ciNPWT
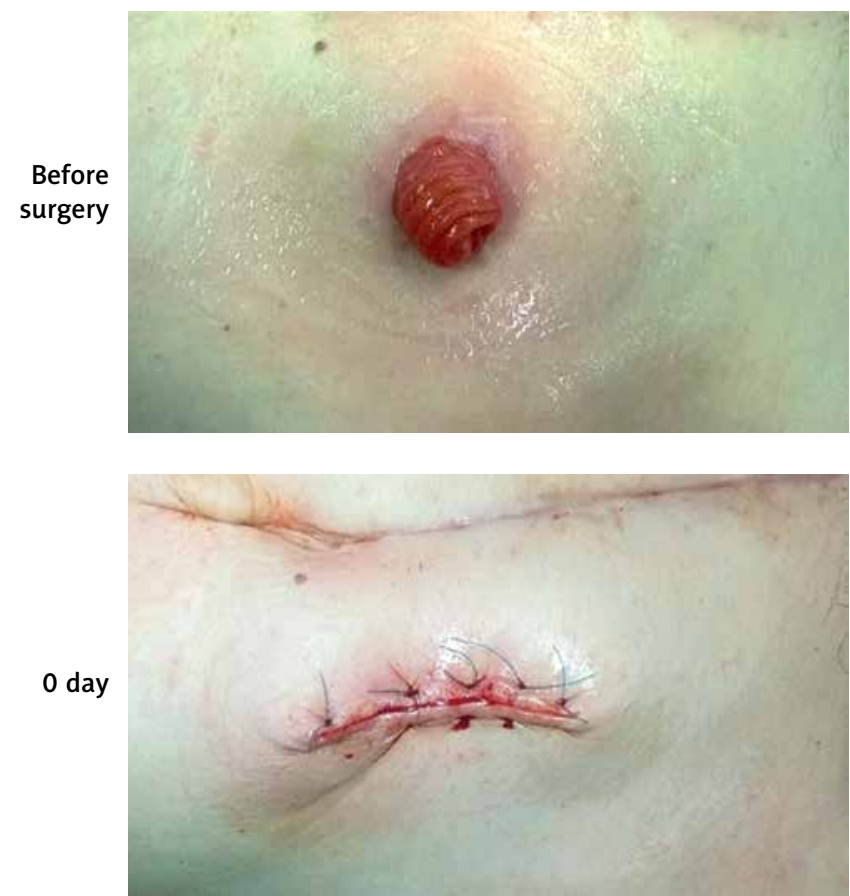

0 day
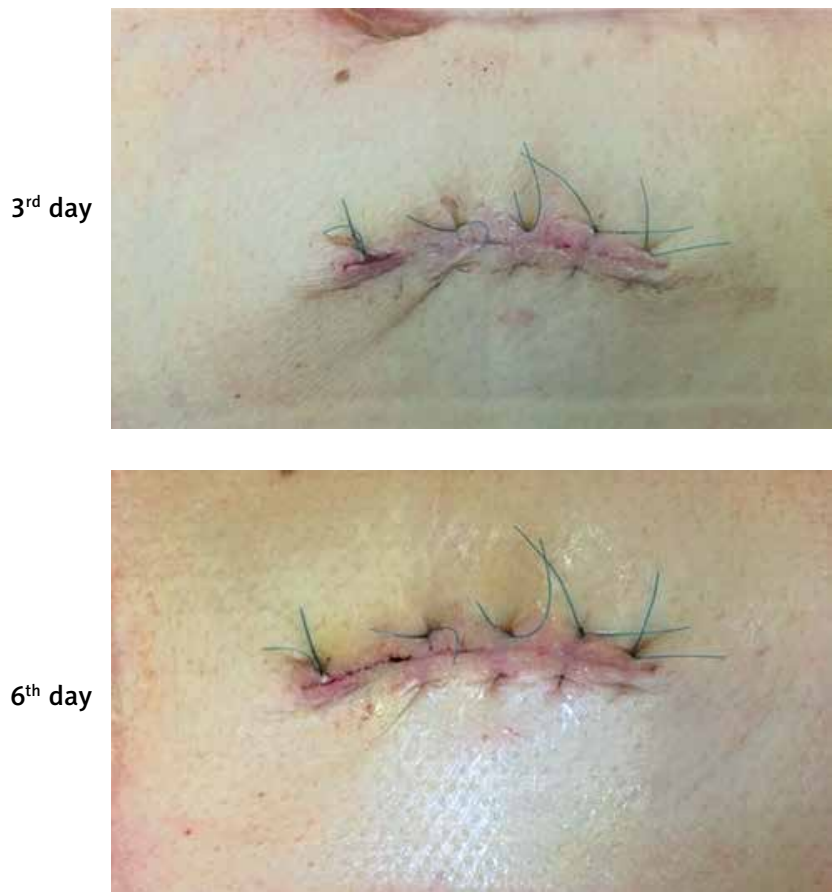

\section{SSD group}
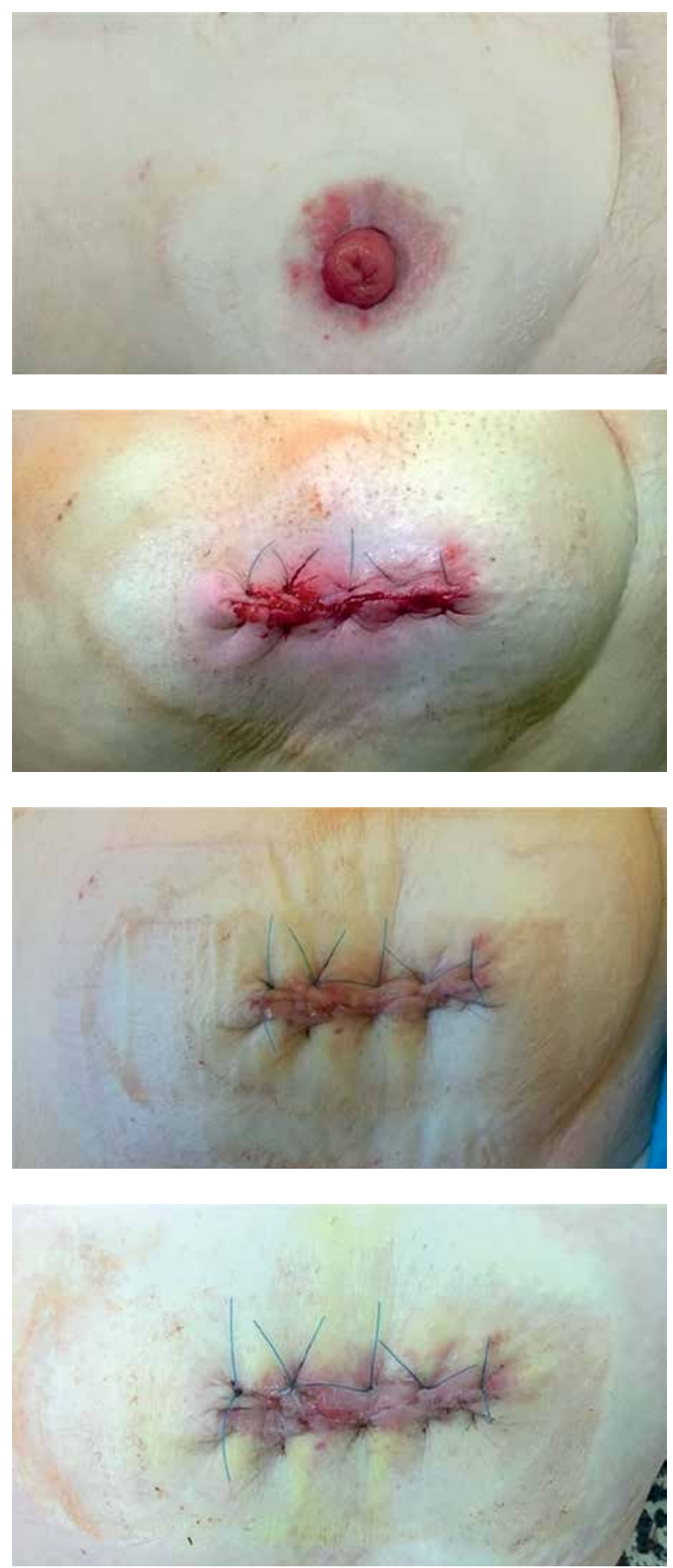

Photo 2. Wound healing on day 0, 3 and 6 after stoma reversal procedure in ciNPWT group (left column) and SSD group (right column). Note the higher wound edema and worse cosmetic effect in the SSD group compared to the ciNPWT group 
on the $1^{\text {st }}($ MdnciNPWT $=4, M d n S S D=5, p=0.027$, $W=51.5)$ and $3^{\text {rd }}$ postoperative days (MdnciNPWT $=2, M d n S S D=4, p=0.014, W=45.5$ ) were significantly lower in the ciNPWT group than in the SSD group. The incidence rate of SSI was reported as $13 \%$ $(2 / 15)$ in the ciNPWT group and $26 \%(4 / 15)$ in the SSD group ( $p=0.651, O R=0.44,95 \% \mathrm{Cl}: 0.03-3.73$ ). All patients in the SSD group who developed SSI presented both local and generalized signs of infection. Culture samples were obtained from the wound bed in SSI patients and an empiric antibiotic regimen was administered, which was switched for another antibiotic regimen if necessary based on antibiotic susceptibility testing results. All 6 patients who developed hematoma or SSI required wound drainage and daily dressing changes, which influenced postponed patients' discharge.

\section{Discussion}

In 1971 Turnbull and Weakly described the first loop ileostomy procedure [19]. Since then, defunctioning stomas have been widely used in many clinical scenarios in order to protect distal intestinal anastomosis. The optimal time for stoma reversal has remained debatable and no firm conclusions have been reached. Most authors recommended stoma closure between 8 and 12 weeks after previous surgery [20]. It is believed that such management allows for recovery after previous bowel resection, reduction of intraabdominal adhesions and appropriate scar remodeling. However, stoma reversal is associated with a high risk of intestinal obstruction, anastomotic leak, enterocutaneous fistulae, stoma site hernias and surgical site infection [21].

Bacterial contamination of the skin surrounding the stoma site or spillage of the intestine contents during stoma closure surgery is the key element resulting in surgical site infection [22]. According to the Centers for Disease Control and Prevention (CDC), the wound after the SR procedure is classified as contaminated [17]. Moreover, due to long-lasting stoma presence, a higher concentration of skin flora not only at the site of the stoma is observed in this group of patients [23]. Although many strategies and techniques of stoma closure have been proposed, the incidence rate of SSI after stoma reversal is still high and ranged from $2 \%$ to $40 \%$ [6, 21-28].

As mentioned above as well as our previous experience with the stoma reversal procedure compli- cated with surgical site infection, we introduced prolonged antibiotic prophylaxis over the $24 \mathrm{~h}$ following the surgery. Moreover, based on the recent consensus on wound antisepsis, one of the key indications for using antiseptics prophylactically is a wound at risk of becoming infected [29].

There is a high need to identify and specify risk factors for SSI after stoma reversal, which may influence quality improvement. Liang et al. based on a single institution cohort revealed four independent predictors for SSI after stoma reversal: a history of fascia dehiscence, thicker subcutaneous fat, presence of a colostomy, and African-American race [26]. However, there was no homogeneity of the analyzed group of patients and they differed in terms of type of stoma and surgical approach, simultaneous hernia repair procedure, type of wound closure and others. Thus, firm conclusions should not be drawn and could not be extrapolated to the ileostomy reversal procedure through the stoma site approach. Recently, Chu et al. investigated the predictors for SSI based on 528 stoma reversal patients, recognizing smoking to be a significant predictor of SSI [30]. Based on the latest evidence-based medicine literature review, the most common risk factors for SSI are: obesity, diabetes mellitus, tobacco smoking, ASA score $\geq 3$, prolong surgical time, and corticosteroid use [31]. Thus, there is an essential need to assess preoperatively patient and procedure dependent risk for developing SSI and modify the pre- and perioperative strategy regarding the type of wound closure and wound dressing used.

The results of our pilot study are comparable to outcomes presented by Poehnert et al. regarding utility of ciNPWT after the stoma reversal procedure [16]. Although the authors revealed that the postoperative wound infection rate was lower in the ciNPWT group, the difference was not statistically significant, which is consistent with our outcomes. However, in our opinion there are some important differences in study design as well as the type of ciNPWT used by Poehnert et al. and ours which may influence the outcomes. Firstly, Poehnert et al. analyzing the risk factors for wound healing disorders found that a statistically significantly greater number of patients had undergone previous chemotherapy in the standard dressing group $(p=0.024)$. In our opinion this is a well-defined risk factor of impairment for wound healing and it might have influenced the better outcomes in the ciNPWT group. 
Secondly, the type of ciNPWT (Prevena incisional wound management system) used by Poehnert et al. is different from the PICO system used in our study. Prevena is composed of Granufoam dressing that may help reduce bacterial colonization. Thirdly, the incorporated canister in the dressing system facilitates greater exudate collection. Fourthly, Prevena delivers negative pressure wound therapy at $-125 \mathrm{~mm} \mathrm{Hg}$, in contrast to the Pico system which generates $-80 \mathrm{~mm} \mathrm{Hg}$. Fifthly, the authors did not present patients' underlying pathologies, which in our opinion may also influence the outcomes.

Based on our experience, we found NPWT to be a useful therapy for abdominal wound management $[32,33]$ as well as colorectal anastomotic leak [34]. The NPWT mechanism of action is multifactorial and includes: drainage of exudate, decreased tissue edema, contraction of the wound edges, stimulation of neoangiogenesis and granulation tissue, increased blood flow in tissue surrounding the wound and others [30-32]. Thus the NPWT dressing creates the optimal conditions and accelerates wound healing. From a practical point of view in the stoma reversal procedure with ciNPWT we suggest application of interrupted sutures with at least $1.0-1.5 \mathrm{~cm}$ intervals. Such application allows for effective absorption of wound excaudate. It is consistent with the recommendations of other authors. Wada et al. recommended preserving the central part of the wound left open using purse string skin closure technique to facilitate wound drainage [35]. It minimizes the blood and serum collection, which may provide an ideal medium for bacterial growth. It was also confirmed that ciNPWT reduced scar thickness formation, and increased tensile strength and mechanical properties of the healed incision line mainly due to increased collagen deposition [36-41].

Introduction of NPWT for the prophylaxis of wound infection in a variety of clinical settings decreased the risk of surgical site infection, wound dehiscence and hematoma/seroma formation. Based on a recent systematic review, decreased incidence rates of SSI, wound dehiscence and hematoma/seroma formation were revealed in the majority of one hundred publications included in the analysis regarding the ciNPWT in various applications [31]. Moreover, a recent meta-analysis showed a 50\% reduction in SSI rate when ciNPWT was used compared to the control group [42]. Application of ciNPWT after open colorectal surgery reduced SSI, as has been confirmed independently by many authors [11-13].

Recently, Wierdak et al. demonstrated in a randomized controlled trial that utility of ciNPWT after ileostomy closure in colorectal cancer patients reduces the incidence of wound healing complications and surgical site infection [43]. Such promising outcomes may have significant implications regarding early stoma reversal surgery, even before adjuvant chemotherapy in colorectal cancer patients as suggested by Kłęk et al. [44]. The results are consistent with the study presented by Okuya et al., who confirmed the usefulness of ciNPWT to prevent SSI [45]. None of fifty consecutive colorectal patients who underwent ileostomy closure developed SSI, seroma or hematoma. However, this is a prospective pilot study and a further comparative study is needed to confirm the outcomes. Comparable results were obtained by Cantero et al., who revealed the usefulness of ciNPWT in reducing SSI in the group of SR patients [46]. The results presented by Uchino et al. differed from the above-mentioned studies, as they did not confirm the efficacy of ciNPWT used after the stoma reversal procedure in ulcerative colitis patients [17]. In our opinion, one explanation may be the time of ciNPWT application. In the cited study, ciNPWT was applied on the first postoperative day and maintained up to 14 days. Potentially, the postponed first ciNPWT application with further maintenance for such a long period of time may lead to bacterial colonization and finally to SSI. Secondly, they used the purse-string suture technique for skin closure, which is associated with longer time of wound healing. Moreover, it is important to note that the type of ciNPWT used may also influence outcomes. Based on recent meta-analyses conducted by Singh et al., analysis of foam dressing versus the control showed statistically significant reduction in SSI rates, whereas no significance was revealed comparing multilayer absorbent dressing versus standard dressing [47]. However, the authors indicated some potential factors which may have influenced the outcomes including: differences in patient selection, type of surgery performed, patient and wound comorbidities, level of negative pressure delivered, dressing interface used, and duration of assessment. Thus, in our opinion, a further comparable study is needed to assess the real potential of different type of ciNPWT in the reduction of postoperative wound-healing complications. Based on our study, we did not confirm with statistical significance the 
superiority of ciNPWT over SSD in regard to SSI rate. One potential explanation may be the small number of patients included in this pilot study. However, this is only a pilot study. Further investigation based on a larger group of patients is needed to establish firmly the benefit for routine application of ciNPWT in stoma reversal patients. Second, the sample group was not homogeneous and the potential risk factors for SSI might vary between patients with different underlying pathologies. Third, no long-term results are presented in the manuscript, as the study is still in progress. Currently, the 30-day follow-up of the patients showed no significant difference between study groups. Based on our previous experience patients who presented with high risk factors for SSI or patients with a high risk procedure may benefit from using ciNPWT. Moreover, the rate of SSI might be underestimated due to varying definitions and considerations. Surgical site infection in inflammatory bowel disease (IBD) patients may be triggered by patient characteristics and comorbidities. Based on a recent expert consensus panel, ciNPWT might be at least suggested or even recommended after the SR procedure due to incision related risk factors (e.g. contamination), operation related risk factors (e.g. open colorectal surgery) and patient related risk factors (e.g. previous corticosteroids usage) [31]. Factors governing colorectal surgical site infection (CSSI) are complicated and multifactorial. Proposed scales estimating the risk of SSI showed that their suitability for clinical practice is still not sufficient [48]. We did not assess the total cost effectiveness of ciNPWT. Although the statistically significant reduction in cost was confirmed in some previous studies, there has been no comprehensive economic analysis of ciNPWT in colorectal surgery. Bonds et al. proved significant total cost savings per patient after colorectal surgery [11]. Chopra et al. reported the economic impact of ciNPWT in high-risk patients after abdominal incisions [49].

\section{Conclusions}

CiNPWT seems not to have a benefit to reduce SSI after the SR procedure. Further investigation is needed to establish firmly the benefit of using ciNPWT in this group of patients.

\section{Conflict of interest}

The authors declare no conflict of interest.

\section{References}

1. Tan WS, Tang CL, Shi L, Eu KW. Meta-analysis of defunctioning stomas in low anterior resection for rectal cancer. Br J Surg 2009; 96: 462-72.

2. van Westreenen HL, Visser A, Tanis PJ, Bemelman WA. Morbidity related to defunctioning ileostomy closure after ileal pouchanal anastomosis and low colonic anastomosis. Int J Colorectal Dis 2012; 27: 49-54.

3. Rubio-Perez I, Leon M, Pastor D, et al. Increased postoperative complications after protective ileostomy closure delay: an institutional study. World J Gastrointest Surg 2014; 6: 169-74.

4. Dindo D, Demartines N, Clavien PA. Classification of surgical complications. A new proposal with evaluation in a cohort of 6336 patients and results of a survey. Ann Surg 2004; 240: 2015-13.

5. Chow A, Tilney HS, Paraskeva P, et al. The morbidity surrounding reversal of defunctioning ileostomies: a systematic review of 48 studies including 6,107 cases. Int J Colorectal Dis 2009; 24: 711-23.

6. Harold DM, Johnson EK, Rizzo JA, Steele SR. Primary closure of stoma site wounds after ostomy takedown. Am J Surg 2010; 199: 621-4.

7. Hsieh MC, Kuo LT, Chi CC, et al. Pursestring closure versus conventional primary closure following stoma reversal to reduce surgical site infection rate: a meta-analysis of randomized controlled trials. Dis Colon Rectum 2015; 58: 808-15.

8. Nie B, Yue B. Biological effects and clinical application of negative pressure wound therapy: a review. J Wound Care 2016; 25: 617-26.

9. Grauhan O, Navasardyan A, Hofmann M, et al. Prevention of poststernotomy wound infections in obese patients by negative pressure wound therapy. J Thorac Cardiovasc Surg 2013; 145: 1387-92.

10. Karlakki SL, Hamad AK, Whittall C, et al. Incisional negative pressure wound therapy dressings (iNPWTd) in routine primary hip and knee arthroplasties: a randomised controlled trial. Bone Joint Res 2016; 5: 328-37.

11. Bonds AM, Novick TK, Dietert JB, et al. Incisional negative pressure wound therapy significantly reduces surgical site infection in open colorectal surgery. Dis Colon Rectum 2013; 56: 1403-8.

12. Blackham AU, Farrah JP, McCoy TP, et al. Prevention of surgical site infections in high-risk patients with laparotomy incisions using negative-pressure therapy. Am J Surg 2013; 205: 647-54.

13. Pellino G, Sciaudone G, Candilio G, et al. Effects of a new pocket device for negative pressure wound therapy on surgical wounds of patients affected with Crohn's disease: a pilot trial. Surg Innov 2014; 21: 204-12.

14. Matatov T, Reddy KN, Doucet LD, et al. Experience with a new negative pressure incision management system in prevention of groin wound infection in vascular surgery patients. J Vasc Surg 2013; 57: 791-5.

15. Pinocy J, Albes JM, Wicke C, et al. Treatment of periprosthetic soft tissue infection of the groin following vascular surgical procedures by means of a polyvinyl alcohol-vacuum sponge system. Wound Repair Regen 2003; 11: 104-9.

16. Poehnert D, Hadeler N, Schrem H, et al. Decreased superficial surgical site infections, shortened hospital stay, and improved 
quality of life due to incisional negative pressure wound therapy after reversal of double loop ileostomy. Wound Repair Regen 2017; 25: 994-1001.

17. Uchino M, Hirose K, Bando T, et al. Randomized controlled trial of prophylactic negative-pressure wound therapy at ostomy closure for the prevention of delayed wound healing and surgical site infection in patients with ulcerative colitis. Dig Surg 2016; 33: 449-54.

18. Culver DH, Horan TC, Gaynes RP, et al. Surgical wound infection rates by wound class, operative procedure, and patient risk in dex. National Nosocomial Infections Surveillance System. Am J Med 1991; 91: 152S-7S.

19. Turnbull RB Jr, Hawk WA, Weakley FL. Surgical treatment of toxic megacolon. lleostomy and colostomy to prepare patients for colectomy. Am J Surg 1971; 122: 325-3.

20. Echazarreta-Gallego E, Elía-Guedea M, Córdoba-Díaz de Las pra E, et al. Defunctioning ileostomy. Is an early closure safe? Int J Colorectal Dis 2016; 31: 771-3.

21. Kaidar-Person O, Person B, Wexner SD. Complications of construction and closure of temporary loop ileostomy. J Am Coll Surg 2005; 201: 759-73.

22. Milanchi S, Nasseri Y, Kidner T, Fleshner P. Wound infection after ileostomy closure can be eliminated by circumferential subcuticular wound approximation. Dis Colon Rectum 2009; 52: 469-74.

23. Cheadle WG. Surgical site infection: still a common problem: comment on "outcomes and predictors of incisional surgical site infection in stoma reversal". JAMA Surg 2013; 148: 183-9.

24. Breckler FD, Rescorla FJ, Billmire DF. Wound infection after colostomy closure for imperforate anus in children: utility of preoperative oral antibiotics. J Pediatr Surg 2010; 45: 1509-13.

25. Lee JT, Marquez TT, Clerc D, et al. Pursestring closure of the stoma site leads to fewer wound infections: results from a multicenter randomized controlled trial. Dis Colon Rectum 2014; 57: 1282-9

26. Liang MK, Li LT, Avellaneda A, et al. Outcomes and predictors of incisional surgical site infection in stoma reversal. JAMA Surg 2013; 148: 183-9.

27. Li LT, Brahmbhatt R, Hicks SC, et al. Prevalence of surgical site infection at the stoma site following four skin closure techniques: a retrospective cohort study. Dig Surg 2014; 31: 73-8.

28. Marquez TT, Christoforidis D, Abraham A, et al. Wound infection following stoma takedown: primary skin closure versus subcuticular pursestring suture. World J Surg 2010; 34: 2877-82.

29. Kramer A, Dissemond J, Kim S, et al. Consensus on wound antisepsis: update 2018. Skin Pharmacol Physiol 2018; 31: 28-58.

30. Chu DI, Schlieve CR, Colibaseanu DT et al. Surgical site infections (SSIS) after stoma reversal (SR): risk factors, implications, and protective strategies. J Gastrointest Surg 2015; 19: 327-34.

31. Willy C, Agarwal A, Andersen CA, et al. Closed incision negative pressure therapy: international multidisciplinary consensus recommendations. Int Wound J 2017; 14: 385-98.

32. Bobkiewicz A, Walczak D, Smoliński S, et al. Management of enteroatmospheric fistula with negative pressure wound therapy in open abdomen treatment: a multicentre observational study. Int Wound J 2017; 14: 255-64.
33. Szmyt K, Łukasz K, Bobkiewicz A, et al. Comparison of the effectiveness of the treatment using standard methods and negative pressure wound therapy (NPWT) in patients treated with open abdomen technique. Pol J Surg 2015; 87: 22-30.

34. Bobkiewicz A, Krokowicz L, Banasiewicz T, Borejsza-Wysocki M. Endoscopic vacuum therapy with instillation (iEVT) - a novel endoscopic concept for colorectal anastomotic leak and perianal complications. Videosurgery Miniinv 2020; 15: 560-6.

35. Wada Y, Miyoshi N, Ohue M, et al. Comparison of surgical techniques for stoma closure: a retrospective study of purse-string skin closure versus conventional skin closure following ileostomy and colostomy reversal. Mol Clin Oncol 2015; 3: 619-22.

36. Orgill DP, Manders EK, Sumpio BE, et al. The mechanisms of action of vacuum assisted closure: more to learn. Surgery 2009; 146: 40-51.

37. Labler L, Rancan M, Mica L, et al. Vacuumassisted closure therapy increases local interleukin- 8 and vascular endothelial growth factor levels in traumatic wounds. I Trauma 2009; 66: 749-57.

38. McNulty A, Spranger I, Courage J, et al. The consistent delivery of negative pressure to wounds using reticulated open cell foam and regulated pressure feedback. Wounds 2010; 22: 114-20.

39. Younan G, Ogawa R, Ramirez M, et al. Analysis of nerve and neuropeptide patterns in vacuum-assisted closure-treated diabetic murine wounds. Plast Reconstr Surg 2010; 126: 87-96.

40. Suh H, Lee AY, Park EJ, Hong JP. Negative pressure wound therapy on closed surgical wounds with dead space: animal study using a swine model. Ann Plast Surg 2016; 76: 717-22.

41. Kilpadi DV, Lessing C, Derrick K. Healed porcine incisions previously treated with a surgical incision management system: mechanical, histomorphometric, and gene expression properties. Aesthetic Plast Surg 2014; 38: 767-78.

42. Semsarzadeh NN, Tadisina KK, Maddox J, et al. Closed incision negative pressure therapy is associated with decreased surgical-site infections: a meta-analysis. Plast Reconstr Surg 2015; 136: 592-602.

43. Wierdak M, Pisarska-Adamczyk M, Major P, et al. Prophylactic negative-pressure wound therapy after ileostomy reversal for the prevention of wound healing complications in colorectal cancer patients: a randomized controlled trial. Tech Coloproctol 2021; 25: 185-93.

44. Kłęk S, Pisarska M, Milian-Ciesielska K, et al. Early closure of the protective ileostomy after rectal resection should become part of the Enhanced Recovery After Surgery (ERAS) protocol: a randomized, prospective, two-center clinical trial. Videosurgery Miniinv 2018; 13: 435-41.

45. Okuya K, Tkemasa I, Tsuruma T, et al. Evaluation of negative-pressure wound therapy for surgical site infections after ileostomy closure in colorectal cancer patients: a prospective multicenter study. Surg Today 2020; 50: 1687-93.

46. Cantero R, Rubio-Perez I, Leon M, et al. Negative-pressure therapy to reduce the risk of wound infection following diverting loop ileostomy reversal: an initial study. Adv Skin Wound Care 2016; 29: 114-8.

47. Singh DP, Gabriel A, Silverman RP, et al. Meta-analysis comparing outcomes of two different negative pressure therapy sys- 
tems in closed incision management. Plast Reconstr Surg Glob Open 2019; 7: e2259.

48. Bergquist JR, Thiels CA, Etzioni DA, et al. Failure of colorecta surgical site infection predictive models applied to an independent dataset: do they add value or just confusion? J Am Coll Surg 2016; 222: 431-8.

49. Chopra K, Gowda AU, Morrow C, et al. The economic impact of closed-incision negative-pressure therapy in high-risk abdominal incisions: a cost utility analysis. Plast Reconstr Surg 2016 137: 1284-9.

Received: 14.12.2020, accepted: 20.02.2021. 